\begin{tabular}{|r|l|}
\hline \multicolumn{2}{|c|}{ Statistica Sinica Preprint No: SS-2020-0359 } \\
\hline Title & $\begin{array}{l}\text { Weighted Rank Estimation for Random-Effects } \\
\text { Monotonic Index Models With Panel Count Data }\end{array}$ \\
\hline Manuscript ID & SS-2020-0359 \\
\hline URL & http://www.stat.sinica.edu.tw/statistica/ \\
\hline DOI & $10.5705 /$ ss.202020.0359 \\
\hline Complete List of Authors & $\begin{array}{l}\text { Tianqing Liu, } \\
\text { Xiaohui Yuan and } \\
\text { Jianguo Sun }\end{array}$ \\
\hline Corresponding Author & Tianqing Liu \\
\hline E-mail & tianqingliu@gmail.com \\
\hline Notice: Accepted version subject to English editing.
\end{tabular}




\title{
Weighted rank estimation for random-effects monotonic index models with panel count data
}

\author{
Tianqing Liu ${ }^{1}$, Xiaohui Yuan ${ }^{2}$ and Jianguo Sun ${ }^{3}$ \\ ${ }^{1}$ Jilin University, ${ }^{2}$ Changchun University of Technology, \\ ${ }^{3}$ University of Missouri
}

\begin{abstract}
Panel count data arise when the study subjects who may experience certain recurrent events are only observed intermittently at discrete examination times. For the situation, in addition to the underlying recurrent event process of interest, there usually exist two other nuisance processes, the observation and follow-up processes, which may be correlated with the recurrent event process of interest. We propose a general class of random-effects monotonic index models for regression analysis of such panel count data. For estimation of regression parameters, a weighted rank (WR) estimation procedure is developed and the consistency and asymptotic normality of the resulting WR estimator are established. A numerical study is conducted and suggests that the proposed methodology works well in practice. An application is also provided.

Key words and phrases: Informative follow-up time, Informative observation process, Random-effects monotonic index models, Panel count data, Weighted rank estimation.
\end{abstract}




\section{Introduction}

Panel count data occur when the study subjects who may experience certain recurrent events are examined only at discrete time points rather than continuously due to cost, feasibility, or other practical considerations (Kalbfleisch \& Lawless, 1985; Thall \& Lachin, 1998; Sun \& Zhao, 2013; Chiou et al., 2019). For the situation, in addition to the underlying recurrent event process of interest, there usually exist two other nuisance processes, the observation and follow-up processes (Wellner \& Zhang, 2000; Lin et al., 2000; Zhang, 2002; Cai \& Schaubel, 2004; Lu, Zhang \& Huang, 2007). Furthermore, they may be correlated with the recurrent event process of interest, leading to so-called informative examination and follow-up times or processes. See Sun \& Zhao (2013) and Chiou et al. (2019) for a comprehensive review and detailed accounts on panel count data.

Many statistical methods have been developed for the analysis of panel count data. Generally, the existing approaches can be broadly classified into three types: pseudo-likelihood estimation, estimating equation approach, and nonparametric estimation. In the pseudo-likelihood procedures, Cox-type models are commonly used and pseudo likelihood function is constructed based on the nonhomogeneous Poisson process assumptions. The likelihood and pseudo-likelihood methods are robust against departure 
from the Poisson assumption as long as the proportional rates model holds (Zhang, 2002; Wellner \& Zhang, 2007; Lu, Zhang \& Huang, 2007; Zhu et al., 2018). The estimating equation approaches are computationally convenient but may be inefficient. Sun \& Wei (2000) and Hu, Sun \& Wei (2003) considered such approaches by modeling cumulative event counts at different time points. Hua \& Zhang (2012) further proposed to improve estimation efficiency of estimating equation approaches through generalized estimating equations.

Kernel smoothing and spline approximation are two popular nonparametric approaches for the analysis of panel count data. For example, Zhao, Tu \& Yu (2018) investigated B-splined pseudo-likelihood method for timevarying coefficients model of panel count data. Wang \& Yu (2019) employed the kernel smoothing to study time varying coefficients panel count model under the assumption of nonhomogeneous Poisson process. However, nonparametric estimation may be difficult for problems with high-dimensional covariates due to the well known 'curse of dimensionality'.

One challenge in the analysis of panel count data is that as mentioned above, the observation process and the follow-up process may be informative about the recurrent event process even after conditioning on available covariates. For example, the patients with higher cancer recurrence rates 
may have more frequent clinical examinations and a longer follow-up time as they may require more medical assessments and attentions (Li et al., 2011; Sun \& Zhao, 2013; Ma \& Sundaram, 2018). Informative examination times and follow-up time are often encountered in panel count data, and falsely treating informative examination times and informative follow-up time as noninformative could result in biased regression coefficient estimation and misleading conclusions.

To account for informative examination times and follow-up time, one way is to specify some joint models for all three processes (Kim, 2006; Sun, Tong \& He, 2007; He, Tong \& Sun, 2009; Buzkova, 2010; Zhao \& Tong, 2011; Zhao, Tong \& Sun, 2013; Zhou et al., 2017; Ma \& Sundaram, 2018; Jiang, Su \& Zhao, 2020). For example, Huang, Wang \& Zhang (2006) and Wang, Ma \& Yan (2013) postulated a frailty proportional rates model for the recurrent event process and left the distributions of the frailty and the possibly correlated examination times unspecified. Chiou et al. (2018) considered a semiparametric accelerated mean model for the recurrent event process and allowed the examination time process and the underlying recurrent event process to be correlated through a shared frailty.

In the following, we propose a general class of random-effects monotonic index models for the recurrent event process of interest in the presence 
of both informative examination times and informative follow-up time. A major advantage of these models is their flexibility and generality as they include many popular models as special cases such as the proportional means model (Lin et al., 2000; Sun \& Wei, 2000; Zhang et al., 2013) and the accelerated mean model (Xu et al., 2017; Chiou et al., 2018). For estimation of regression parameters, we will develop a weighted rank (WR) estimation method, which is insensitive to the choice of observation process and censoring mechanism.

Many authors have developed various rank estimation methods for different types of regression models. For example, Han (1987) proposed distribution-free maximum rank correlation estimators for generalized regression models. Cavanagh \& Sherman (1998) proposed monotone rank estimators for monotonic linear index models. Lin et al. (2017) provided a maximum rank correlation estimator for random-effects transformation models when the random-effects distribution is symmetric. Liu, Yuan \& Sun (2021a) proposed a weighted rank estimator for nonparametric transformation models with nonignorable missing data. One can refer to Abrevaya (1999), Khan \& Tamer (2007), Wang \& Chiang (2019) and Liu, Yuan \& Sun (2021b, 2021c) for applications of rank estimation methods in other contexts. To the best of our knowledge, there is no rank estimation proce- 
dure available for the analysis of the recurrent event process in the presence of both informative examination times and informative follow-up time.

The remaining of the paper is organized as follows. In Section 2, we introduce the random-effects monotonic index model and present the proposed WR estimators of regression parameters. Also the consistency and asymptotic normality of the WR estimators are established and a random weighting resampling scheme is provided for approximating the distribution of the WR estimators. Some simulations results are presented in Section 3 and suggest that the proposed method works well for practical situations. In Section 4, the approach is applied to a set of real panel count data and some concluding remarks are given in Section 5. The proofs of the asymptotic results are postponed to the section S1 of Supplementary Materials.

\section{Weighted rank estimation for random-effects monotonic index}

\section{models}

In this section, we will first describe a class of random-effects monotonic index models and then present the proposed weighted rank estimation procedure.

\subsection{Random-effects monotonic index models}

Consider a study involving $N$ subjects who may experience recurrent events. For subject $i \in\{1, \cdots, N\}$, let $M_{i}(t)$ represent the cumulative 
number of events that have occurred before time $t, 0 \leq t \leq \tau$, where $\tau$ is a known constant time point. For subject $i$, suppose that there is a $(p+1)$-dimensional vector $W_{i}$ of covariates whose effects on $M_{i}(t)$ are of main interest. For $i=1, \cdots, N$, let $K_{i}$ denote the number of observation times for the $i$ th subject and we allow $K_{i}$ to take value 0 , which means that there are no observations for the $i$ th subject. Even if the follow time for each subject is $\tau$, the counting process $M_{i}(t)$ would only be observed only at finite time points $T_{i(1)}<\cdots<T_{i\left(K_{i}\right)}$, which are order statistics of $\left\{T_{i 1}, \cdots, T_{i K_{i}}\right\}$. Naturally, not every subject can be followed until $\tau$. Let $C_{i}$ be the follow-up time for the $i$ th subject. Then, $M_{i}\left(T_{i k}\right)$ cannot be observed when $C_{i}<T_{i k}<\tau$. That is, we only observe panel count data given by

$$
\left\{\left(T_{i k}, M_{i k}=M_{i}\left(T_{i k}\right), W_{i}^{\top}, C_{i}\right)^{\top}: T_{i k} \leq C_{i}, k=1, \cdots, K_{i}, i=1, \cdots, N\right\}
$$

Let $Z_{i}$ be a $q$-dimensional random vector of latent variables that is independent of $W_{i}$. We assume that given $\left\{W_{i}, Z_{i}\right\}$ the conditional mean function of $M_{i}(t)$ follows random-effects monotonic index models:

$$
E\left\{M_{i}(t) \mid W_{i}, Z_{i}\right\}=\mu\left(W_{i}^{\top} \boldsymbol{\beta}^{*}, Z_{i}, t\right)
$$

where $\left\|\boldsymbol{\beta}^{*}\right\|=1$ and for fixed $Z_{i}$ and $t, \mu\left(W_{i}^{\top} \boldsymbol{\beta}^{*}, Z_{i}, t\right)$ is an unspecified strictly increasing function of $W_{i}^{\top} \boldsymbol{\beta}^{*}$. If $M_{i}(t)$ is a Poisson process, equation $(22)$ is a conventional model for the mean of a Poisson variable on the 
interval $(0, \tau)$. In this paper, we are interested in making inferences about $\boldsymbol{\beta}^{*}$ in model $\left([2.2)\right.$ without assuming that $M_{i}(\cdot)$ is a homogeneous or nonhomogeneous Poisson process.

We denote $\left\{H_{i}(t), t \geq 0\right\}$ as the observation process or examination time process, which is a point process

$$
H_{i}(t)=\sum_{k=1}^{K_{i}} I\left(T_{i k} \leq t\right),
$$

representing the cumulative visit numbers up to time $t$. Throughout this paper, for any real numbers $\left\{a_{i}\right\}_{i \geq 1}$, we set $\sum_{k=1}^{0} a_{i} \equiv 0$. Thus, if $K_{i}=0$, we have $H_{i}(t) \equiv 0$. Let

$$
\begin{aligned}
O_{i} & =O_{i}(\cdot)=\left(W_{i}^{\top}(\cdot), Z_{i}^{\top}(\cdot), C_{i}(\cdot), M_{i}(\cdot), H_{i}(\cdot)\right)^{\top} \\
& =\left(W_{i}^{\top}, Z_{i}^{\top}, C_{i}, M_{i}(\cdot), H_{i}(\cdot)\right)^{\top}, \quad i=1, \cdots, N,
\end{aligned}
$$

be independent copies of $O=O(\cdot)=\left(W^{\top}(\cdot), Z^{\top}(\cdot), C(\cdot), M(\cdot), H(\cdot)\right)^{\top}=$ $\left(W^{\top}, Z^{\top}, C, M(\cdot), H(\cdot)\right)^{\top}$, where $W=\left(X_{1}, \cdots, X_{p+1}\right)^{\top}$. We assume that $C, H(\cdot)$ and $M(\cdot)$ are conditionally independent given $\{W, Z\}$. For the observation process and follow-up time, we assume that,

$$
\begin{aligned}
& E\{H(t) \mid W, Z\}=\varsigma(W) \nu(Z, t), \\
& P(C>t \mid W, Z)=\kappa_{1}(W, t) \kappa_{2}(Z, t),
\end{aligned}
$$

where $\varsigma(\cdot), \nu(\cdot, \cdot), \kappa_{1}(\cdot, \cdot)$ and $\kappa_{2}(\cdot, \cdot)$ are unspecified functions such that 
$\varsigma(\cdot) \geq 0, \nu(\cdot, \cdot) \geq 0,0 \leq \kappa_{1}(\cdot, \cdot) \kappa_{2}(\cdot, \cdot) \leq 1$, and for all fixed $\{Z, W\}$ $\partial \nu(Z, t) / \partial t \geq 0$ and $\partial\left\{\kappa_{1}(W, t) \kappa_{2}(W, t)\right\} / \partial t \leq 0$.

Remark 1. Let $H(t)=\sum_{k=1}^{K} I\left(T_{k} \leq t\right)$. Suppose $K$ and $\left\{T_{k}: k \geq 1\right\}$ are conditionally independent given $\{W, Z\}, E(K \mid W, Z)=\varsigma(W) \nu_{1}(Z)$ and $E\left\{I\left(T_{k} \leq t\right) \mid W, Z\right\}=\nu_{2}(Z, t)$ for all $k \geq 1$. Then, it is easy to verify that

$$
E\{H(t) \mid W, Z\}=\varsigma(W) \nu_{1}(Z) \nu_{2}(Z, t)
$$

which shows that $(\mathbb{2 . 4})$ is satisfied with $\varsigma(W)$ and $\nu(Z, t)=\nu_{1}(Z) \nu_{2}(Z, t)$.

Example 1. Assume that given $\{W, Z\}, K$ has a Poisson distribution with mean $\varsigma(W) \nu_{1}(Z)>0$, and $T_{k}, k \geq 1$, are independent and identically distributed with distribution function $\{F(t)\}^{\sigma(Z)}$, where $\sigma(z)>0$ for $z \in \mathbb{R}^{q}$ and $F(t)$ is a distribution function such that $f(t)=d F(t) / d t>0$ for $t>0$. Under these assumptions, $E\{H(t) \mid W, Z\}=\varsigma(W) \nu(Z, t)$, where $\nu(Z, t)=\nu_{1}(Z) \nu_{2}(Z, t)$ and $\nu_{2}(Z, t)=\{F(t)\}^{\sigma(Z)}$.

Remark 2. Suppose $G(C)=G_{1}\left(\zeta_{1}, Z\right) \wedge G_{2}\left(\zeta_{2}, W\right)$, where $\zeta_{1}, \zeta_{2}, Z$ and $W$ are independent, $G(\cdot)$ is an unknown increasing function, $G_{1} \wedge G_{2}=$ $\min \left(G_{1}, G_{2}\right), G_{1}(\cdot, \cdot)$ and $G_{2}(\cdot, \cdot)$ are unknown functions. Then, (2.5) is satisfied.

Example 2. Assume that, $C=\Lambda_{a} \wedge \Lambda_{b}$, where $\Lambda_{a}$ and $\Lambda_{b}$ are conditionally independent given $\{W, Z\} . \Lambda_{a}$ and $\Lambda_{b}$ follow exponential distributions with 
means $1 / \lambda_{1}(W)>0$ and $1 / \lambda_{2}(Z)>0$, respectively. Under these assumptions, $P(C>t \mid W, Z)=\kappa_{1}(W, t) \kappa_{2}(Z, t)$, where $\kappa_{1}(W, t)=\exp \left\{-\lambda_{1}(W) t\right\}$ and $\kappa_{2}(Z, t)=\exp \left\{-\lambda_{2}(Z) t\right\}$

Remark 3. The frailty proportional rates models (Huang, Wang \& Zhang, 2006; Wang, Ma \& Yan, 2013) and semiparametric accelerated mean models (Chiou et al., 2018) are important cases of the proposed random-effects monotonic index models. Let $Z$ denote a latent nonnegative frailty variable, whose distribution is unspecified and satisfies that $E(Z \mid W)=1$. The frailty proportional rates model specifies the mean of the event process $M(t)$, given the frailty variable $Z$ and covariate $W$, as $\mu_{1}\left(W^{\top} \boldsymbol{\beta}^{*}, Z, t\right)=$ $Z \Lambda(t) \exp \left(W^{\top} \boldsymbol{\beta}^{*}\right)$, where $\Lambda(\cdot)$ is a completely unspecified baseline mean function. The semiparametric accelerated mean model assumes that the recurrent event process $M(t)$, conditioning on the latent nonnegative frailty variable $Z$ and covariate $W$, has the mean function $\mu_{2}\left(W^{\top} \boldsymbol{\beta}^{*}, Z, t\right)=$ $Z_{i} \Lambda_{0}\left\{t \exp \left(W^{\top} \boldsymbol{\beta}^{*}\right)\right\}$, where $\Lambda_{0}(t)=\int_{0}^{t} \lambda_{0}(u) d u$ and $\lambda_{0}(u)$ is an unspecified, absolutely continuous baseline rate function.

Remark 4. Motivated by the frailty proportional rates models and semiparametric accelerated mean models, it is possible to relax the independence assumption of $Z$ and $W$. Specifically, assume that given $\{W, Z\}$, $C, H(\cdot)$ and $M(\cdot)$ are conditionally independent, and the conditional mean 
function of $M(t)$ follows the following special random-effects monotonic index models: $E\{M(t) \mid W, Z\}=g(Z) \pi\left(W^{\top} \boldsymbol{\beta}^{*}, t\right)$, where $\left\|\boldsymbol{\beta}^{*}\right\|=1, g(Z)$ is an unspecified nonnegative function of $Z$ such that $E\{g(Z) \mid W\}=1$ and for fixed $t, \pi\left(W^{\top} \boldsymbol{\beta}^{*}, t\right)$ is an unspecified strictly increasing function of $W^{\top} \boldsymbol{\beta}^{*}$. For the observation process and follow-up time, assume that, $E\{H(t) \mid W, Z\}=\varsigma(W) \sigma(Z) \nu(t)$ and $P(C>t \mid W, Z)=\kappa(W, t)$, where $\varsigma(\cdot)$, $\sigma(\cdot), \nu(\cdot)$, and $\kappa(\cdot, \cdot)$ are unspecified functions such that $\varsigma(\cdot) \geq 0, \sigma(\cdot) \geq 0$, $E\{\sigma(Z) \mid W\}=1, \nu(\cdot) \geq 0,0 \leq \kappa(\cdot, \cdot) \leq 1, \partial \nu(t) / \partial t \geq 0$, and for fixed $W$ $\partial \kappa(W, t) / \partial t \leq 0$. Then, under certain mild conditions, the consistency of the WR estimator $\hat{\boldsymbol{\beta}}$ (See subsection 2.2 ) to $\boldsymbol{\beta}^{*}$ still holds.

\subsection{Weighted rank estimators}

To construct a consistent rank regression estimator of $\boldsymbol{\beta}^{*}$ analogous to the maximum rank correlation estimator in Han (1987) or the monotone rank estimator in Cavanagh \& Sherman (1998), we wish to construct a function $\Psi\left(O_{i}, O_{j}\right)$ which satisfies the property

$$
\begin{aligned}
& E\left\{\Psi\left(O_{i}, O_{j}\right) \mid W_{i}, W_{j}\right\} \geq E\left\{\Psi\left(O_{j}, O_{i}\right) \mid W_{i}, W_{j}\right\} \\
& \text { if and only if } W_{i}^{\top} \boldsymbol{\beta}^{*}>W_{j}^{\top} \boldsymbol{\beta}^{*} .
\end{aligned}
$$


For the problem at hand with panel count data, we set

$$
\begin{aligned}
& U_{i j}=\sum_{k=1}^{K_{i}} I\left(T_{i k} \leq C_{i} \wedge C_{j}\right) M_{i k}, \quad V_{i j}=\sum_{l=1}^{K_{j}} I\left(T_{j l} \leq C_{i} \wedge C_{j}\right), \\
& \Psi\left(O_{i}, O_{j}\right)=Y_{i j}=U_{i j} V_{i j}, \quad i, j=1, \cdots, N
\end{aligned}
$$

Here, $Y_{i j}=0$ if $K_{i} K_{j}=0$. Let $\Theta=\left\{\boldsymbol{\beta} \in \mathbb{R}^{p+1}:\|\boldsymbol{\beta}\|=1\right\}$. The proposed WR estimator of $\boldsymbol{\beta}^{*}$ is defined as

$$
\hat{\boldsymbol{\beta}}=\arg \max _{\boldsymbol{\beta} \in \Theta} Q_{N}(\boldsymbol{\beta})
$$

where

$$
Q_{N}(\boldsymbol{\beta})=\frac{1}{N^{2}-N} \sum_{i \neq j}^{N} Y_{i j} I\left(W_{i}^{\boldsymbol{\top}} \boldsymbol{\beta}>W_{j}^{\boldsymbol{\top}} \boldsymbol{\beta}\right) .
$$

Note that $\left\{Q_{N}(\boldsymbol{\beta}): \boldsymbol{\beta} \in \Theta\right\}$ is a U-process of order 2. The consistency and asymptotic normality of $\hat{\boldsymbol{\beta}}$ are given by the following theorems.

Theorem 1. Under the conditions C0-C5 in the Appendix, we have $\hat{\boldsymbol{\beta}} \stackrel{p}{\longrightarrow}$ $\boldsymbol{\beta}^{*}$ as $N \rightarrow \infty$.

Note that the maximization of $Q_{N}(\boldsymbol{\beta})$ with respect to $\boldsymbol{\beta}$ in (‥9) is subject to the constraint $\|\boldsymbol{\beta}\|=1$. Following Liu, Yuan \& Sun (2021a), we derive the asymptotic distribution of $\hat{\boldsymbol{\beta}}$ by first reparameterizing $\boldsymbol{\beta}^{*}=$ $\boldsymbol{\beta}\left(\boldsymbol{\vartheta}^{*}\right)=\boldsymbol{\beta}\left(\boldsymbol{\theta}^{*}, \alpha^{*}\right)=:\left(\boldsymbol{\theta}^{* \top}, \alpha^{*} \sqrt{1-\left\|\boldsymbol{\theta}^{*}\right\|^{2}}\right)^{\top}$, where $\boldsymbol{\vartheta}^{*}=\left(\boldsymbol{\theta}^{* \top}, \alpha^{*}\right)^{\top}$ and $\boldsymbol{\theta}^{*}=\left(\theta_{1}^{*}, \cdots, \theta_{p}^{*}\right)^{\top}$. Let $\operatorname{sgn}(\cdot)$ denote the $\operatorname{sign}$ function, i.e, $\operatorname{sgn}(u)=$ $I(u>0)-I(u<0)$ for $u \in \mathbb{R}$. Obviously, we can write $\boldsymbol{\vartheta}^{*}=\boldsymbol{\vartheta}\left(\boldsymbol{\beta}^{*}\right)=$ : 
$\left(\beta_{1}^{*}, \cdots, \beta_{p}^{*}, \operatorname{sgn}\left(\beta_{p+1}^{*}\right)\right)^{\top}$, where $\alpha^{*}=\operatorname{sgn}\left(\beta_{p+1}^{*}\right)$ and $\theta_{j}^{*}=\beta_{j}^{*}, j=1, \cdots, p$.

Define

$$
\hat{\boldsymbol{\vartheta}}=\left(\hat{\boldsymbol{\theta}}^{\top}, \hat{\alpha}\right)^{\top}=\arg \max _{\boldsymbol{\vartheta} \in \Upsilon} Q_{N}\{\boldsymbol{\beta}(\boldsymbol{\vartheta})\}
$$

where $\Upsilon=\{\boldsymbol{\vartheta}(\boldsymbol{\beta}): \boldsymbol{\beta} \in \Theta\}$. Then, it is easy to see that $\hat{\boldsymbol{\theta}}=\left(\hat{\beta}_{1}, \cdots, \hat{\beta}_{p}\right)^{\top}$ and $\hat{\alpha}=\operatorname{sgn}\left(\hat{\beta}_{p+1}\right)$. From Theorem $\square$ and the condition $\mathrm{C} 4$ in the Appendix, $\hat{\boldsymbol{\vartheta}}$ is a consistent estimator of $\boldsymbol{\vartheta}^{*}$.

Theorem 2. Define

$Q(\boldsymbol{\beta})=E\left[\Psi\left(O_{1}, O_{2}\right) I\left(W_{1}^{T} \boldsymbol{\beta}>W_{2}^{T} \boldsymbol{\beta}\right)\right], \quad A(\boldsymbol{\vartheta})=-\partial^{2} Q\{\boldsymbol{\beta}(\boldsymbol{\vartheta})\} / \partial \boldsymbol{\theta} \partial \boldsymbol{\theta}^{T}$, where $\boldsymbol{\vartheta}=\left(\boldsymbol{\theta}^{T}, \alpha\right)^{T}$. Let $U$ be a normal random vector with mean $0_{p \times 1}$ and covariance matrix $\Sigma=\operatorname{cov}\left\{h\left(O_{i}, \boldsymbol{\vartheta}^{*}\right)\right\}$, where

$$
\begin{aligned}
& h\left(O_{i}, \boldsymbol{\vartheta}\right)=A^{-1}(\boldsymbol{\vartheta}) \partial b_{i}(\boldsymbol{\vartheta}) / \partial \boldsymbol{\theta}, \\
& b_{i}(\boldsymbol{\vartheta})=E\left[a_{i j}(\boldsymbol{\vartheta})+a_{j i}(\boldsymbol{\vartheta})-2 E\left\{a_{i j}(\boldsymbol{\vartheta})\right\} \mid O_{i}\right] \\
& a_{i j}(\boldsymbol{\vartheta})=\Psi\left(O_{i}, O_{j}\right)\left[I\left\{W_{i}^{T} \boldsymbol{\beta}(\boldsymbol{\vartheta})>W_{j}^{T} \boldsymbol{\beta}(\boldsymbol{\vartheta})\right\}\right. \\
& \left.-I\left\{W_{i}^{T} \boldsymbol{\beta}\left(\boldsymbol{\theta}^{*}, \alpha\right)>W_{j}^{T} \boldsymbol{\beta}\left(\boldsymbol{\theta}^{*}, \alpha\right)\right\}\right] .
\end{aligned}
$$

If the conditions C0-C6 in the Appendix are satisfied, we have $N^{1 / 2}(\hat{\boldsymbol{\vartheta}}-$ $\left.\boldsymbol{\vartheta}^{*}\right)=N^{1 / 2}\left(\left(\hat{\boldsymbol{\theta}}-\boldsymbol{\theta}^{*}\right)^{T},\left(\hat{\alpha}-\alpha^{*}\right)\right)^{T} \stackrel{d}{\longrightarrow}\left(U^{\boldsymbol{T}}, 0\right)^{\boldsymbol{T}}$ as $N \rightarrow \infty$. Define $B(\boldsymbol{\vartheta})=$ $\partial \boldsymbol{\beta}(\boldsymbol{\vartheta}) / \partial \boldsymbol{\vartheta}^{T}$. Then, as $N \rightarrow \infty, N^{1 / 2}\left(\hat{\boldsymbol{\beta}}-\boldsymbol{\beta}^{*}\right)=N^{1 / 2}\left\{\boldsymbol{\beta}(\hat{\boldsymbol{\vartheta}})-\boldsymbol{\beta}\left(\boldsymbol{\vartheta}^{*}\right)\right\} \stackrel{d}{\longrightarrow}$ $B\left(\boldsymbol{\vartheta}^{*}\right)\left(U^{T}, 0\right)^{T}$, which is a normal random vector with mean $0_{(p+1) \times 1}$ and covariance matrix $\Omega=\operatorname{cov}\left\{B\left(\boldsymbol{\vartheta}^{*}\right)\left(U^{T}, 0\right)^{T}\right\}$. 


\subsection{Coordinate-wise algorithm}

In this section, by following Wu \& Stefanski (2015) and Liu, Yuan \& Sun (2021a), we will present a coordinate-wise optimization algorithm to optimize the objective function $Q_{N}(\boldsymbol{\beta})$. The idea of such algorithm is to maximize one coordinate at a time with the other fixed. Define

$$
\ell_{k}(\gamma \mid \boldsymbol{\zeta}, \boldsymbol{\eta})=\sum_{(i, j) \in \mathscr{S}} p_{i j} I\left\{\left(\boldsymbol{\zeta}^{\top}, \gamma, \boldsymbol{\eta}^{\top}\right)\left(W_{i}-W_{j}\right)<0\right\}, \quad k=1, \cdots, p+1,
$$

where $\boldsymbol{\zeta}^{\top}=\left(\zeta_{1}, \cdots, \zeta_{k-1}\right), \boldsymbol{\eta}^{\top}=\left(\eta_{1}, \cdots, \zeta_{p+1-k}\right), p_{i j}=Y_{j i}$ and $\mathscr{S}=$ $\left\{(i, j): p_{i j} \neq 0\right\}$. Note that we set $\boldsymbol{\zeta}=\emptyset$ if $k=1$ and $\boldsymbol{\eta}=\emptyset$ if $k=p+1$.

To this end, we can write $\ell_{k}(\gamma \mid \boldsymbol{\zeta}, \boldsymbol{\eta})$ as

$$
\begin{aligned}
\ell_{k}(\gamma \mid \boldsymbol{\zeta}, \boldsymbol{\eta}) & =\sum_{(i, j) \in \mathscr{S}} p_{i j} I\left(a_{i j, k} \gamma<b_{i j, k}\right) \\
& =\sum_{(i, j) \in \mathscr{S}} p_{i j}\left\{I\left(\gamma<c_{i j, k}\right) I\left(a_{i j, k}>0\right)+I\left(\gamma>c_{i j, k}\right) I\left(a_{i j, k}<0\right)\right\} \\
& =\sum_{l=1}^{L} p_{l}\left\{I\left(\gamma<c_{l, k}\right) I\left(a_{l, k}>0\right)+I\left(\gamma>c_{l, k}\right) I\left(a_{l, k}<0\right)\right\},(2.12)
\end{aligned}
$$

where $c_{i j, k}=b_{i j, k} / a_{i j, k}, a_{i j, k}=X_{i k}-X_{j k}, b_{i j, k}=-\sum_{l=1}^{k-1}\left(X_{i l}-X_{j l}\right) \zeta_{l}-$ $\sum_{l=k+1}^{p+1}\left(X_{i l}-X_{j l}\right) \eta_{l-k}$ and

$$
\begin{aligned}
& \left\{\left(a_{i j, k}, b_{i j, k}, c_{i j, k}, p_{i j, k}\right):(i, j) \in \mathscr{S}\right\} \\
= & \left\{\left(a_{l, k}, b_{l, k}, c_{l, k}, p_{l, k}\right): c_{1, k}<\cdots<c_{L, k}, L=|\mathscr{S}|\right\} .
\end{aligned}
$$

Here, for ease of presentation, the dependence of $\left(b_{i j, k}, c_{i j, k}\right)$ and $\left(b_{l, k}, c_{l, k}\right)$ on $(\boldsymbol{\zeta}, \boldsymbol{\eta})$ is suppressed. Note that the objective function $\ell_{k}(\gamma \mid \boldsymbol{\zeta}, \boldsymbol{\eta})$ is just a 
piecewise constant function with cut-off values $\left\{c_{l}\right\}_{l=1}^{L}$. Furthermore, Liu, Yuan \& Sun (2021a) showed that

$$
\begin{array}{r}
\ell_{k}\left(c_{s+1, k} \mid \boldsymbol{\zeta}, \boldsymbol{\eta}\right)=\ell_{k}\left(c_{s, k} \mid \boldsymbol{\zeta}, \boldsymbol{\eta}\right)-p_{s+1} I\left(a_{s+1, k}>0\right)+p_{s} I\left(a_{s, k}<0\right), \\
s=1, \cdots, L-1 .
\end{array}
$$

Using ([.]3), the values of $\left\{\ell_{k}\left(c_{l, k} \mid \boldsymbol{\zeta}, \boldsymbol{\eta}\right)\right\}_{l=1}^{L}$ can be easily obtained. Thus, $\ell_{k}(\gamma \mid \boldsymbol{\zeta}, \boldsymbol{\eta})$ is very easy to maximize. It follows that

$$
\hat{\gamma}_{k}(\boldsymbol{\zeta}, \boldsymbol{\eta})=\arg \max _{\gamma \in \mathbb{R}} \ell_{k}(\gamma \mid \boldsymbol{\zeta}, \boldsymbol{\eta})=\arg \max _{\gamma \in\left\{c_{1, k}, \cdots, c_{L}, k\right.} \ell_{k}(\gamma \mid \boldsymbol{\zeta}, \boldsymbol{\eta})
$$

To this end, the proposed coordinate optimization algorithm is given by

1. Set initial value $\boldsymbol{\beta}^{(0)}=\left(\beta_{1}^{(0)}, \cdots, \beta_{p+1}^{(0)}\right)^{\top}$;

2. Given $\boldsymbol{\beta}^{(m)}=\left(\beta_{1}^{(m)}, \cdots, \beta_{p+1}^{(m)}\right)^{\top}$, for $k=1, \cdots, p+1$, compute

$$
\beta_{k}^{(m+1)}=\hat{\gamma}_{k}\left(\left(\beta_{1}^{(m+1)}, \cdots, \beta_{k-1}^{(m+1)}\right)^{\top},\left(\beta_{k+1}^{(m)}, \cdots, \beta_{p+1}^{(m)}\right)^{\top}\right)
$$

where $\hat{\gamma}_{k}(\boldsymbol{\zeta}, \boldsymbol{\eta})$ is defined in (2.14). Then, set $\boldsymbol{\beta}^{(m+1)}=\left(\beta_{1}^{(m+1)}, \cdots, \beta_{p+1}^{(m+1)}\right)^{\mathrm{T}}$

3. Repeat step 2 till $\left|Q_{N}\left(\boldsymbol{\beta}^{(m+1)}\right)-Q_{N}\left(\boldsymbol{\beta}^{(m)}\right)\right|<10^{-6}$. Denote the final value of $\boldsymbol{\beta}$ by $\boldsymbol{\beta}^{(\infty)}$;

4. Set $\hat{\boldsymbol{\beta}}=\boldsymbol{\beta}^{(\infty)} /\left\|\boldsymbol{\beta}^{(\infty)}\right\|$.

Obviously, the proposed coordinate-wise optimization algorithm guarantees a monotone increasing of $Q_{N}(\boldsymbol{\beta})$ at each iteration step in a very efficient manner. That is, we always have $Q_{N}\left(\boldsymbol{\beta}^{(m+1)}\right) \geq Q_{N}\left(\boldsymbol{\beta}^{(m)}\right), m=1,2, \cdots$. 
To apply theorem $\nabla$ to make inference about $\boldsymbol{\beta}^{*}$, we should estimate the limiting covariance matrix $\Omega$ of $\hat{\boldsymbol{\beta}}$. Inspired by the method of Jin, Ying \& Wei (2001), we develop a resampling scheme to approximate the distribution of $\hat{\boldsymbol{\beta}}$, particularly its covariance matrix. Let $\lambda_{1}, \cdots, \lambda_{N}$ be independent and identically distributed exponential random variables with mean 1, i.e., $\operatorname{Exp}(1)$. The resampling WR estimator of $\boldsymbol{\beta}^{*}$ is defined as

$$
\hat{\boldsymbol{\beta}}^{*}=\arg \max _{\boldsymbol{\beta} \in \Theta} Q_{N}^{*}(\boldsymbol{\beta})
$$

where

$$
Q_{N}^{*}(\boldsymbol{\beta})=\frac{1}{N^{2}-N} \sum_{i \neq j}^{N} \lambda_{i} \lambda_{j} Y_{i j} I\left(W_{i}^{\top} \boldsymbol{\beta}>W_{j}^{\top} \boldsymbol{\beta}\right) .
$$

Then we have the following proposition.

Proposition 1. Under the conditions CO-C6 in the Appendix, given $\left\{O_{i}, i=\right.$ $1, \cdots, N\}, N^{1 / 2}\left(\hat{\boldsymbol{\beta}}^{*}-\hat{\boldsymbol{\beta}}\right) \stackrel{d}{\longrightarrow} N\left(0_{(p+1) \times 1}, \Omega\right)$ as $N \rightarrow \infty$, which is the limiting distribution of $N^{1 / 2}\left(\hat{\boldsymbol{\beta}}-\boldsymbol{\beta}^{*}\right)$.

The proof of proposition $⿴$ is similar to those of Jin, Ying \& Wei (2001) and thus omitted. Theoretically, the distribution of $N^{1 / 2}\left(\hat{\boldsymbol{\beta}}-\boldsymbol{\beta}^{*}\right)$ can be approximated by the resampling distribution of $N^{1 / 2}\left(\hat{\boldsymbol{\beta}}^{*}-\hat{\boldsymbol{\beta}}\right)$ given the data $\left\{O_{i}, i=1, \cdots, N\right\}$. In practice, for $b=1, \cdots, B$, we produce $\hat{\boldsymbol{\beta}}_{b}^{*}$ by solving (2.15) with random weights $\left(\lambda_{b 1}, \cdots, \lambda_{b N}\right)$ in $([2.16)$ while holding the data $\left\{O_{i}, i=1, \cdots, N\right\}$ at their observed values. The distribution of 
$\hat{\boldsymbol{\beta}}$ can then be approximated by the empirical distribution of $\left\{\hat{\boldsymbol{\beta}}_{b}^{*}\right\}_{b=1}^{B}$. The asymptotic covariance matrix $\Omega$ can be estimated by $\hat{\Omega}=N B^{-1} \sum_{b=1}^{B}\left(\hat{\boldsymbol{\beta}}_{b}^{*}-\right.$ $\left.\overline{\boldsymbol{\beta}}^{*}\right)\left(\hat{\boldsymbol{\beta}}_{b}^{*}-\overline{\boldsymbol{\beta}}^{*}\right)^{\top}$ with $\overline{\boldsymbol{\beta}}^{*}=B^{-1} \sum_{b=1}^{B} \hat{\boldsymbol{\beta}}_{b}^{*}$. For $j=1, \cdots, p+1$, the $100 \times$ $(1-\alpha) \%$ confidence interval of $\beta_{j}^{*}$ is given by $\left[\hat{\beta}_{j}-N^{-1 / 2} \hat{\omega}_{j j}^{1 / 2} C_{1-\alpha / 2}, \hat{\beta}_{j}+\right.$ $\left.N^{-1 / 2} \hat{\omega}_{j j}^{1 / 2} C_{1-\alpha / 2}\right]$, where $\hat{\omega}_{j j}$ is the $(j, j)$ element of $\hat{\Omega}$ and $C_{1-\alpha / 2}$ is the $1-\alpha / 2$ quantile of standard normal distribution.

\section{Simulation Study}

Now we present some results obtained from a simulation study conducted to investigate the performance of the WR estimator $\hat{\boldsymbol{\beta}}$ proposed above and compare it to several other estimators given in the literature for the same problem. Additional results for the complicated simulation settings are given in the Section S2 of Supplementary Materials.

In the study, we assume that the latent variable $Z_{i}$ follows a gamma distribution with parameters $(2,2)$ and $K_{i}$ follows the uniform distribution over $\{3,4,5,6\}$. We set $\tau=8$ and $C_{i}=\min \left(C_{i}^{*}, \tau\right)$, where $C_{i}^{*}$ follows the uniform distribution over $[1,10]$. Given $K_{i}$, the observation times $T_{i 1}, \cdots, T_{i K_{i}}$ are independent and identically distributed with uniform distribution over $\left(0, C_{i}\right)$. Let $W_{i}=\left(X_{i 1}, X_{i 2}\right)^{\top}$, where $X_{1 i}$ is a standard normal random variable, $X_{i 2}$ is an exponential random variable with mean 1 . Given $W_{i}, Z_{i}, K_{i}$ 
and $\left(T_{i 1}, \cdots, T_{i K_{i}}\right)$, we generate $M_{i k}=M\left(T_{i k}\right)$ by using the formula

$$
M_{i}\left(T_{i k}\right)=M_{i}\left(T_{i 1}\right)+\left\{M_{i}\left(T_{i 2}\right)-M_{i}\left(T_{i 1}\right)\right\}+\cdots+\left\{M_{i}\left(T_{i k}\right)-M_{i}\left(T_{i, k-1}\right)\right\}
$$

and assuming that $M_{i}(t)-M_{i}(s)$ follows the Poisson distribution with mean

$$
\mu\left(W_{i}^{\top} \boldsymbol{\beta}^{*}, Z_{i}, t\right)-\mu\left(W_{i}^{\top} \boldsymbol{\beta}^{*}, Z_{i}, s\right)
$$

On the random-effects monotonic index model, we consider the following four conditional mean functions $(\mathrm{CMF})$ :

(a) $\mu\left(W_{i}^{\top} \boldsymbol{\beta}^{*}, Z_{i}, t\right)=\operatorname{tg}\left(Z_{i}\right) \exp \left(W_{i}^{\top} \boldsymbol{\beta}^{*}\right)$

(b) $\mu\left(W_{i}^{\top} \boldsymbol{\beta}^{*}, Z_{i}, t\right)=t\left\{g\left(Z_{i}\right)+\exp \left(W_{i}^{\top} \boldsymbol{\beta}^{*}\right)+1\right\}$;

(c) $\mu\left(W_{i}^{\boldsymbol{\top}} \boldsymbol{\beta}^{*}, Z_{i}, t\right)=t\left\{g\left(Z_{i}\right)+\exp \left(W_{i}^{\boldsymbol{\top}} \boldsymbol{\beta}^{*}\right)\right\}$

(d) $\mu\left(W_{i}^{\top} \boldsymbol{\beta}^{*}, Z_{i}, t\right)=\operatorname{tg}\left(Z_{i}\right)+\exp \left(W_{i}^{\top} \boldsymbol{\beta}^{*}\right)$,

where $\boldsymbol{\beta}^{*}=\left(\beta_{1}^{*}, \beta_{2}^{*}\right)^{\top}$ and $g(Z)=\sqrt{Z}$ or $g(Z)=\exp (-Z / 5)$.

As mentioned above, several methods have been proposed in the literature for the problem discussed here under the simpler model (a). In the study, we compared the proposed WR estimator to four such estimators, the estimating equation-based estimator given in Sun \& Wei (2000), the maximum pseudo-likelihood estimator given in Zhang (2002), the maximum likelihood estimator given in Lu, Zhang \& Huang (2007) and the augmented estimating equation-based estimator given in Wang, Ma \& Yan 
(2013). In the following, they will be referred to as EE, MPL, ML and AEE estimators, respectively, and are determined by using the $\mathrm{R}$ function panelReg in the SPEF package (Chiou et al., 2019). The results given below are based on $N=100$ or 200 and $B=200$ with 1000 replications.

The results on the comparison of the proposed WR estimator to the four existing estimators are given in Tables $1-2$ with $\boldsymbol{\beta}^{*}=(-1,1)^{\top} / \sqrt{2}$ and $N=100$ and 200, and here we calculated both the empirical bias (Bias) and the mean square errors (MSEs) for each of the five estimators. As seen above, the WR estimator is always unbiased and also stable cross all the cases. In comparison, the four other estimators seem to be unbiased under model (a) but to have some biases under models (b), (c) and (d). Also the proposed WR estimator appears to have smaller MSE than the EE, MPL, ML and AEE estimators under models (b), (c) and (d). Moreover, MPL, ML and WR estimators perform similarly according to bias and MSE under model (a). In other words, as expected, the WR estimator applies to more general situations than the existing estimators.

Tables 3-4 presents the results obtained on the proposed WR estimator under the simulation settings described above with $\boldsymbol{\beta}^{*}=(-1,1)^{\top} / \sqrt{2}$ and $N=100$ and 200. They include the sample standard deviation of the estimates (SD), the average of the estimated standard deviations (ESD), and 
the $95 \%$ empirical coverage probability $(\mathrm{CP})$. It can be seen from the tables that the ESD appears to be close to the SD and thus appropriate. In addition, the results on the $\mathrm{CP}$ suggest that the normal approximation to the distribution of the WR estimator seems to be reasonable and as expected, the results became better when the sample size increased. We also presented additional simulation results under other setups in the supplementary material and obtained similar results.

\section{Data analysis}

In this section, we apply the proposed WR estimation procedure to a well-known bladder cancer data set (Andrews \& Herzberg, 1985, p.250-260; Sun \& Wei, 2000). A total of 116 patients with bladder tumors were randomized to three treatment groups: placebo, pyridoxine, and thiotepa. The main objective is to assess the treatment effects on reducing the occurrence rate of bladder tumors. Since the patients were only observed periodically, only the numbers of the occurrences of bladder tumors between observation times are available. That is, only panel count data are observed on the underlying bladder tumor occurrence process. In addition to the treatment indicators, there exists another covariate, the initial number of bladder tumors.

To apply the proposed estimation procedure, let $M(t)$ denote the total 
number of the recurrences of bladder tumors up to time $t$ and assume that $M(t)$ can be described by the index model (2). Define $X_{1}$ to be the initial number of tumors, $X_{2}=1$ if the patient was given the thiotepa treatment and zero otherwise, and $X_{3}=1$ if the patient was given the pyridoxine treatment and zero otherwise.

Table 5 presents the estimated covariate effects with $B=200$, including the WR estimates, the estimated standard deviation (ESD) and the $p$-values for testing $\beta_{j}^{*}=0, j=1,2,3$.

For comparison, we also include in the table the results based on the other four estimation procedures discussed in the previous section. One can see from Table 5 that the five methods basically give similar conclusions and all suggest that the thiotepa treatment has some positive effect of lowering the bladder tumor recurrence rate. In comparison, the pyridoxine treatment does not seem to have any effect in reducing the bladder tumor

rate. Although the results from different approaches are similar, the WR method indicate some stronger thiotepa treatment effect than the others.

\section{Conclusions and discussions}

In this paper, we proposed a class of of random-effects monotonic index models for regression analysis of panel count data, which arise commonly in many fields. One challenge for the problem is that, in addition to 
the underlying recurrent event process of interest, one has to deal with two nuisance processes, the observation process and the follow-up process, which may be correlated with the recurrent event process. The proposed random-effects monotonic index models include many popular models as special cases such as the proportional means model and the accelerated mean model. For estimation, a WR estimation procedure was developed and the asymptotic properties of the resulting estimators of regression parameters were established. In addition, the numerical results indicated that the proposed approach applies to more general situations than the existing methods.

In this paper, our focus has been on the estimation of regression parameters and sometimes one may be also interested in estimating the mean function $\mu$ in (2.2) as well as the relationship between the recurrent event process of interest and the observation process. It is apparent that a new method has to be developed for solving these issues. Another direction for future research is that when there exist several correlated recurrent event processes and one observes multivariate panel count data (Sun \& Zhao, 2013). For the situation, one key issue is how to model the correlation between these processes. Of course, one could choose to leave the correlation structure arbitrary if only the marginal effects are of interest. 


\section{Supplementary Materials}

Supplementary material contains the proofs of Theorems 1-2 and additional simulation results.

Acknowledgements. On behalf of all authors, the corresponding author states that there is no conflict of interest.

\section{References}

Abrevaya, J. (1999). Rank estimation of a transformation model with observed truncation. Econom. J., 2, 292-305.

Buzkova, P. (2010). Panel count data regression with informative observation times. Int. J. Biostat., 6, Article 30 .

Cai, J. and Schaubel, D. E. (2004). Analysis of recurrent event data. Handbook of statistics, 23, $603-623$.

Cavanagh, C. and Sherman, R. P. (1998). Rank estimators for monotonic index models. J. Econometrics, 84(2), 351-381.

Chiou, S. H., Xu, G., Yan, J. and Huang C.-Y. (2018). Semiparametric estimation of the accelerated mean model with panel count data under informative examination times. Biometrics, 74, 944-953.

Chiou, S. H., Huang, H.-Y., Xu, G. and Yan, J. (2019). Semiparametric regression analysis of 
panel count data: a practical review. Int. Stat. Rev., 87(1), 24-43.

Han, A. K. (1987). Nonparametric analysis of a generalized regression model: The maximum rank correlation estimator. J. Econometrics, 35(2-3), 303-316.

He, X., Tong, X. and Sun, J. (2009). Semiparametric analysis of panel count data with correlated observation and follow-up times. Lifetime Data Anal., 15(2), 177-196.

Hu, X., Sun, J. and Wei, L.-J. (2003). Regression parameter estimation from panel counts. Scand. J. Stat., 30, 25-43.

Hua, L. and Zhang, Y. (2012). Spline-based semiparametric projected generalized estimating equation method for panel count data. Biostatistics, 13, 440-454.

Huang, C.-Y., Wang, M.-C. and Zhang, Y. (2006). Analysing panel count data with informative observation times. Biometrika, 93, 763-775.

Jiang, H., Su, W. and Zhao, X. (2020). Robust estimation for panel count data with informative observation times and censoring times. Lifetime Data Anal., 26, 65-84.

Jin, Z., Ying, Z. and Wei, L. J. (2001). A simple resampling method by perturbing the minimand. Biometrika, 88(2), 381-390.

Kalbfleisch, J. and Lawless, J. F. (1985). The analysis of panel data under a markov assumption. J. Am. Statist. Assoc., 80, 863-871.

Khan, S. and Tamer, E. (2007). Partial rank estimation of duration models with general forms of censoring. J. Econometrics, 136(1), 251-280. 


\section{REFERENCES}

Kim, Y.-J. (2006). Analysis of panel count data with dependent observation times. Comm.

Statist. Simulation Comput., 35, 983-990.

Li, N., Park, D.-H., Sun, J. and Kim, K. (2011). Semiparametric transformation models for multivariate panel count data with dependent observation process. Canad. J. Statist., 39, $458-474$.

Lin, D., Wei, L., Yang, I. and Ying, Z. (2000). Semiparametric regression for the mean and rate functions of recurrent events. J. R. Statist. Soc. B, 62(4), 711-730.

Lin, Y., Luo, Y., Xie, S. and Chen, K. (2017). Robust rank estimation for transformation models with random effects. Biometrika, 104(4), 971-986.

Liu, T., Yuan, X. and Sun, J. (2021a). Weighted rank estimation for nonparametric transformation models with nonignorable missing data. Comput. Statist. Data Anal., 153, 107061.

Liu, T., Yuan, X. and Sun, J. (2021b). Weighted rank estimation for nonparametric transformation models with doubly truncated data. J. Korean Statist. Soc., 50, 1-24.

Liu, T., Yuan, X. and Sun, J. (2021c). Weighted rank estimation of nonparametric transformation models with case-1 and case-2 interval-censored failure time data. J. Nonparametr. Stat., 33, 225-248.

Lu, M., Zhang, Y. and Huang, J. (2007). Estimation of the mean function with panel count data using monotone polynomial splines. Biometrika, 94, 1-14.

Ma, L. and Sundaram, R. (2018). Analysis of gap times based on panel count data with informative observation times and unknown start time. J. Am. Statist. Assoc., 113, 294-305. 
Sherman, R. (1993). The limiting distribution of the maximum rank correlation estimator. Econometrica, 61(1), 123-137.

Sherman, R. (1994). Maximal inequalities for degenerate U-processes with applications to optimization estimators. Ann. Statist., 22(1), 439-459.

Sun, J., Tong, X. and He, X. (2007). Regression analysis of panel count data with dependent observation times. Biometrics, 63, 1053-1059.

Sun, J. and Wei, L. J. (2000). Regression analysis of panel count data with covariate-dependent observation and censoring times. J. R. Statist. Soc. B, 62, 293-302.

Sun, J. and Zhao, X. (2013). The Statistical Analysis of Panel Count Data, Springer Science+Business Inc.

Xu, G., Chiou, S. H., Huang, C.-Y., Wang, M.-C. and Yan, J. (2017). Joint scale-change models for recurrent events and failure time. J. Am. Statist. Assoc., 112, 794-805.

Thall, P. F. and Lachin, J. M. (1988). Analysis of recurrent events: Nonparametric methods for random-interval count data. J. Am. Statist. Assoc., 83, 339-347.

Wang, S.-H. and Chiang, C.-T. (2019). Maximum partial-rank correlation estimation for lefttruncated and right-censored survival data. Statist. Sinica, 29, 2141-2161.

Wang, X., Ma, S. and Yan, J. (2013). Augmented estimating equations for semiparametric panel count regression with informative observation times and censoring time. Statist. Sinica, 23, $359-381$. 


\section{REFERENCES}

Wu, Y. and Stefanski, L. A. (2015). Automatic structure recovery for additive models. Bometrika, 102, 381-395.

Wang, Y. and Yu, Z. (2019). A kernel regression model for panel count data with time-varying coefficients. arXiv:1903.10233v1 [math.ST]

Wellner, J. A. and Zhang Y. (2000). Two estimators of the mean of a counting process with panel count data. Ann. Statist., 28, 779-814.

Zhang, Y. (2002). A semiparametric pseudolikelihood estimation method for panel count data. Biometrika, 89, 39-48.

Zhang, H., Zhao, H., Sun, J., Wang, D. and Kim, K. (2013). Regression analysis of multivariate panel count data with an informative observation process. J. Multivariate Anal., 119, $71-80$.

Zhao, X. and Tong, X. (2011). Semiparametric regression analysis of panel count data with informative observation times. Comput. Statist. Data Anal., 55, 291-300.

Zhao, H., Tu, W. and Yu, Z. (2018). A nonparametric time-varying coefficient model for panel count data. J. Nonparametr. Stat., 30(3), 640-661.

Zhou, J., Zhang, H., Sun, L. and Sun, J. (2017). Joint analysis of panel count data with an informative observation process and a dependent terminal event. Lifetime Data Anal., 23, $560-584$

Zhu, L., Zhang, Y., Li, Y., Sun, J. and Robison, L. L. (2018). A semiparametric likelihood based method for regression analysis of mixed panel count data. Biometrics, 74, 488-497. 
Center for Applied Statistical Research and School of Mathematics, Jilin University, Changchun 130012, Jilin, China

E-mail: tianqingliu@gmail.com

School of Mathematics and Statistics, Changchun University of Technology, Changchun 130012, Jilin, China

E-mail: yuanxh@ccut.edu.cn

Department of Statistics, University of Missouri, USA

E-mail: sunj@missouri.edu

(Received ???? 20??; accepted ???? 20??)

\section{Appendix.}

In this appendix, we will give the conditions for the proof of the asymptotic results in

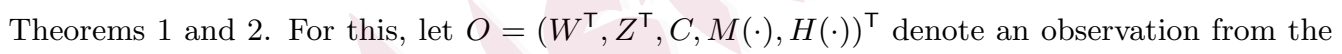
distribution $P$ on the set $\mathcal{O} \subseteq \mathbb{R}^{p+1} \times \mathbb{R}^{q} \times \mathbb{R}_{+} \times D(0, \infty) \times D(0, \infty)$, where $D(0, \infty)$ is the space of real-valued cadlag functions on $[0, \infty)$. For each $o=o(\cdot)=(w, z, c, m(\cdot), h(\cdot))^{\top}$ in $\mathcal{O}$ and each $\boldsymbol{\beta}$ in $\Theta$, define

$$
\varrho(o, \boldsymbol{\beta})=E\left\{\Psi(O, o) I\left(W^{\top} \boldsymbol{\beta}>w^{\top} \boldsymbol{\beta}\right)\right\}+E\left\{\Psi(o, O) I\left(w^{\top} \boldsymbol{\beta}>W^{\top} \boldsymbol{\beta}\right)\right\},
$$

where $w=\left(x_{1}, \cdots, x_{p+1}\right)^{\top}$ and $\Psi\left(O_{1}, O_{2}\right)$ is defined in (区8). Write $\nabla_{m}$ for the $m$ th partial 


\section{REFERENCES}

derivative operator of the function $\varrho(z, \boldsymbol{\beta})$ with respect to $\boldsymbol{\beta}=\left(\beta_{1}, \cdots, \beta_{p+1}\right)^{\top} \in \mathbb{R}^{p+1}$, and let

$$
\left|\nabla_{k}\right| \varrho(o, \boldsymbol{\beta})=\sum_{i_{1}, \cdots, i_{k} \in\{1, \cdots, p+1\}}\left|\frac{\partial^{k}}{\partial \beta_{i_{1}} \cdots \partial \beta_{i_{k}}} \varrho(o, \boldsymbol{\beta})\right| .
$$

Let $\mathcal{W}, \mathcal{Z}$ and $\mathcal{C}$ denote the supports of $W, Z$ and $C$, respectively. To establish the asymptotic properties, we require the following conditions.

C0 (a) $O_{i}=\left(W_{i}^{\top}, Z_{i}^{\top}, C_{i}, M_{i}(\cdot), H_{i}(\cdot)\right)^{\top}, i=1, \cdots, N$ are independent copies of

$$
O=\left(W^{\top}, Z^{\top}, C, M(\cdot), H(\cdot)\right)^{\top}
$$

(b) $C, M(\cdot)$ and $H(\cdot)$ are conditionally independent given $\{W, Z\}$; (c) $W$ and $Z$ are independent; (d) $E\{M(t) \mid W, Z\}=\mu\left(W^{\top} \boldsymbol{\beta}^{*}, Z, t\right)$ and for fixed $\{Z, t\}, \mu\left(W^{\top} \boldsymbol{\beta}^{*}, Z, t\right)$ is an unspecified strictly increasing function of $W^{\top} \boldsymbol{\beta}^{*} ;(\mathrm{e}) E\{H(t) \mid W, Z\}=\varsigma(W) \nu(Z, t)$, $P(C>t \mid W, Z)=\kappa_{1}(W, t) \kappa_{2}(Z, t)$, where $\varsigma(\cdot), \nu(\cdot), \kappa_{1}(\cdot, \cdot)$ and $\kappa_{2}(\cdot, \cdot)$ are unspecified functions such that $\varsigma(\cdot) \geq 0, \nu(\cdot, \cdot) \geq 0,0 \leq \kappa_{1}(\cdot, \cdot) \kappa_{2}(\cdot, \cdot) \leq 1$, and for all fixed $Z \in \mathcal{Z}$, $W \in \mathcal{W}$ and $t>0, \partial \nu(Z, t) / \partial t \geq 0$ and $\partial\left\{\kappa_{1}(W, t) \kappa_{2}(W, t)\right\} / \partial t \leq 0 ;$

C1 Assume that for all $\left(w_{1}, w_{2}\right) \in \mathcal{W} \times \mathcal{W}, \varsigma\left(w_{1}\right) \varsigma\left(w_{2}\right)>0$. For all $\left(w_{1}, w_{2}\right) \in \mathcal{W} \times$ $\mathcal{W}$, there exist compact subsets $\mathcal{Z}_{w_{1}, w_{2}} \subseteq \mathcal{Z}$ and $\mathcal{C}_{w_{1}, w_{2}} \subseteq \mathcal{C}$ such that (a) $P(Z \in$ $\left.\mathcal{Z}_{w_{1}, w_{2}}\right) \mathscr{L}\left(\mathcal{C}_{w_{1}, w_{2}}\right)>0$, where $\mathscr{L}(\cdot)$ is the Lebesgue measure on $\mathbb{R}$; (b) for all $r \in \mathcal{Z}_{w_{1}, w_{2}}$, $(s, r) \in \mathcal{Z}_{w_{1}, w_{2}} \times \mathcal{Z}_{w_{1}, w_{2}}$ and $a \in \mathcal{C}_{w_{1}, w_{2}}$,

$$
\begin{aligned}
& \frac{\partial\left\{1-\kappa_{1}\left(w_{1}, a\right) \kappa_{2}(s, a) \kappa_{1}\left(w_{2}, a\right) \kappa_{2}(r, a)\right\}}{\partial a}>0, \\
& \int_{0}^{a} \frac{\partial \nu(r, t)}{\partial t} d t>0
\end{aligned}
$$


$\mathrm{C} 2$ The set $\mathcal{W}$ is not contained in any proper linear subspace of $\mathbb{R}^{p+1}$;

C3 The vector of covariates, $W=\left(X_{1}, \cdots, X_{p+1}\right)^{\top}$, is of full rank, and there exists $j \in$ $\{1, \cdots, p+1\}$ such that $X_{j}$ has an everywhere-positive Lebesgue density conditional on $X_{-j}=\left(X_{1}, \cdots, X_{j-1}, X_{j+1}, \cdots, X_{p+1}\right)^{\top}$

C4 The unknown parameter $\boldsymbol{\beta}^{*}=\left(\beta_{1}^{*}, \cdots, \beta_{p+1}^{*}\right)^{\top}$, where $\boldsymbol{\beta}^{*} \in \Theta, \beta_{j}^{*} \neq 0$, and $j$ is defined in condition $\mathrm{C} 3$; without loss of generality, assume that $j=p+1$ in conditions $\mathrm{C} 3-\mathrm{C} 4$;

C5 $E\left(Y_{12}^{2}\right)=E\left\{\Psi^{2}\left(O_{1}, O_{2}\right)\right\}<+\infty$.

C6 (a) Let $\mathcal{B}$ denote a neighborhood of $\boldsymbol{\beta}^{*}$. For each $o$, all mixed second partial derivatives of $\varrho(o, \boldsymbol{\beta})$ exist on $\mathcal{B}$. There is a function $\omega(o)$ such that $E\{\omega(O)\}<+\infty$ and for all $o \in \mathcal{O}$ and $\boldsymbol{\beta}$ in $\mathcal{B}$,

$$
\left\|\nabla_{2} \varrho(o, \boldsymbol{\beta})-\nabla_{2} \varrho\left(o, \boldsymbol{\beta}^{*}\right)\right\| \leq \omega(o)\left\|\boldsymbol{\beta}-\boldsymbol{\beta}^{*}\right\|
$$

(b) $E\left\{\left\|\nabla_{1} \varrho\left(O, \boldsymbol{\beta}^{*}\right)\right\|^{2}\right\}<+\infty$; (c) $E\left\{\left|\nabla_{2}\right| \varrho\left(O, \boldsymbol{\beta}^{*}\right)\right\}<+\infty$;

(d) $\left.E\left\{\partial^{2} \varrho\left\{O, \boldsymbol{\beta}\left(\boldsymbol{\theta}, \alpha^{*}\right)\right\} / \partial \boldsymbol{\theta} \partial \boldsymbol{\theta}^{\top}\right\}\right|_{\boldsymbol{\theta}=\boldsymbol{\theta}^{*}}$ is negative definite.

Most of the above conditions are assumed for rank estimation of a standard random-

effects monotonic index model. Additional conditions are on the observation process, censoring mechanism and the distribution assumptions on $\left(C, W^{\top}, Z^{\top}\right)^{\top}$. C0 defines the structure which generates the observations. In particular, $\mathrm{C} 0(\mathrm{~d})$ contains the key monotonicity assumption. Conditions C0-C5 guarantee the identifiability of $\boldsymbol{\beta}^{*}$. Specifically, conditions C0-C5 ensure that 


\section{REFERENCES}

$Q(\boldsymbol{\beta})=E\left\{Q_{N}(\boldsymbol{\beta})\right\}$ is uniquely maximized over $\Theta$ at $\boldsymbol{\beta}^{*}$, which can be proved by verifying that the inequality in ([2.6]) holds if and only if $\boldsymbol{\beta}=\boldsymbol{\beta}^{*}$.

From C0, we can write

$$
P\left(C_{1} \wedge C_{2}>a \mid W_{1}, W_{2}, Z_{1}, Z_{2}\right)=\kappa_{1}\left(W_{1}, a\right) \kappa_{2}\left(Z_{1}, a\right) \kappa_{1}\left(W_{2}, a\right) \kappa_{2}\left(Z_{2}, a\right)
$$

Thus, the conditional density of $C_{1} \wedge C_{2}$ given $\left\{W_{1}, W_{2}, Z_{1}, Z_{2}\right\}$ can be written as

$$
f_{C_{1} \wedge C_{2}}\left(a \mid W_{1}, W_{2}, Z_{1}, Z_{2}\right)=\frac{\partial\left\{1-\kappa_{1}\left(W_{1}, a\right) \kappa_{2}\left(Z_{1}, a\right) \kappa_{1}\left(W_{2}, a\right) \kappa_{2}\left(Z_{2}, a\right)\right\}}{\partial a} .
$$

Observing ( $A .3)$, ( $A .7$ ) in $C 1$ is easily satisfied. Note that (A.2) in C1 is easily satisfied if we assume $\partial \nu(r, t) / \partial t>0$ for all $r \in \mathcal{Z}$ and $t>0$. For the consistency proof, condition C5 can be weakened to a finite first moment for $Y_{12}$, but the normality result requires a finite second moment. Conditions C6 contains standard regularity conditions sufficient to support an argument based on a Taylor expansion of $\varrho\left\{o ; \boldsymbol{\beta}\left(\boldsymbol{\theta}, \alpha^{*}\right)\right\}$ about $\boldsymbol{\theta}$. 
Table 1: Simulation results under four different conditional mean functions with $g(Z)=\sqrt{Z}$. Bias: empirical bias; MSE: mean square error; CMF: conditional mean function.

\begin{tabular}{|c|c|c|c|c|c|c|}
\hline \multirow[b]{2}{*}{$N$} & \multirow[b]{2}{*}{$\mathrm{CMF}$} & \multirow[b]{2}{*}{ Estimator } & \multicolumn{2}{|c|}{$\beta_{1}^{*}$} & \multicolumn{2}{|c|}{$\beta_{2}^{*}$} \\
\hline & & & Bias & MSE & Bias & MSE \\
\hline \multirow[t]{20}{*}{100} & $\mathrm{a}$ & WR & 0.0093 & 0.0082 & 0.0004 & 0.0044 \\
\hline & & $\mathrm{AEE}$ & 0.0010 & 0.0061 & -0.0017 & 0.0039 \\
\hline & & ML & 0.0014 & 0.0063 & -0.0026 & 0.0039 \\
\hline & & MPL & 0.0017 & 0.0056 & -0.0029 & 0.0037 \\
\hline & & $\mathrm{EE}$ & -0.0032 & 0.0075 & -0.0077 & 0.0073 \\
\hline & $\mathrm{b}$ & WR & 0.0191 & 0.0118 & 0.0056 & 0.0073 \\
\hline & & AEE & 0.3368 & 0.1165 & -0.2431 & 0.0638 \\
\hline & & ML & 0.3363 & 0.1162 & -0.2428 & 0.0637 \\
\hline & & MPL & 0.3449 & 0.1219 & -0.2524 & 0.0680 \\
\hline & & $\mathrm{EE}$ & 0.4133 & 0.1763 & -0.3122 & 0.1050 \\
\hline & $\mathrm{c}$ & WR & 0.0151 & 0.0085 & 0.0051 & 0.0057 \\
\hline & & $\mathrm{AEE}$ & 0.2683 & 0.0747 & -0.1886 & 0.0388 \\
\hline & & ML & 0.2679 & 0.0745 & -0.1886 & 0.0388 \\
\hline & & MPL & 0.2775 & 0.0796 & -0.1975 & 0.0420 \\
\hline & & $\mathrm{EE}$ & 0.3524 & 0.1305 & -0.2538 & 0.0715 \\
\hline & d & WR & 0.0066 & 0.0080 & -0.0024 & 0.0047 \\
\hline & & AEE & 0.1105 & 0.0172 & -0.0745 & 0.0098 \\
\hline & & ML & 0.1104 & 0.0174 & -0.0745 & 0.0099 \\
\hline & & MPL & 0.1164 & 0.0181 & -0.0785 & 0.0101 \\
\hline & & $\mathrm{EE}$ & 0.1797 & 0.0385 & -0.1211 & 0.0209 \\
\hline
\end{tabular}




\section{REFERENCES}

Table 1 (continued): Simulation results under four different conditional mean functions with $g(Z)=\sqrt{Z}$. Bias: empirical bias; MSE: mean square error; CMF: conditional mean function.

\begin{tabular}{|c|c|c|c|c|c|c|}
\hline \multirow[b]{2}{*}{$N$} & \multirow[b]{2}{*}{$\mathrm{CMF}$} & \multirow[b]{2}{*}{ Estimator } & \multicolumn{2}{|c|}{$\beta_{1}^{*}$} & \multicolumn{2}{|c|}{$\beta_{2}^{*}$} \\
\hline & & & Bias & MSE & Bias & MSE \\
\hline \multirow[t]{20}{*}{200} & $\mathrm{a}$ & WR & 0.0051 & 0.0050 & 0.0001 & 0.0021 \\
\hline & & $\mathrm{AEE}$ & -0.0009 & 0.0035 & 0.0056 & 0.0033 \\
\hline & & ML & 0.0006 & 0.0032 & -0.0026 & 0.0023 \\
\hline & & MPL & 0.0011 & 0.0028 & -0.0022 & 0.0021 \\
\hline & & $\mathrm{EE}$ & -0.0026 & 0.0036 & 0.0010 & 0.0031 \\
\hline & $\mathrm{b}$ & WR & 0.0042 & 0.0044 & -0.0010 & 0.0031 \\
\hline & & $\mathrm{AEE}$ & 0.3253 & 0.1080 & -0.2248 & 0.0538 \\
\hline & & ML & 0.3250 & 0.1079 & -0.2247 & 0.0538 \\
\hline & & MPL & 0.3333 & 0.1131 & -0.2334 & 0.0575 \\
\hline & & $\mathrm{EE}$ & 0.4130 & 0.1733 & -0.3072 & 0.0981 \\
\hline & $\mathrm{c}$ & WR & 0.0075 & 0.0036 & 0.0031 & 0.0026 \\
\hline & & $\mathrm{AEE}$ & 0.2553 & 0.0670 & -0.1711 & 0.0315 \\
\hline & & ML & 0.2551 & 0.0669 & -0.1711 & 0.0315 \\
\hline & & MPL & 0.2641 & 0.0714 & -0.1793 & 0.0342 \\
\hline & & $\mathrm{EE}$ & 0.3495 & 0.1249 & -0.2495 & 0.0651 \\
\hline & d & WR & 0.0053 & 0.0053 & -0.0002 & 0.0024 \\
\hline & & $\mathrm{AEE}$ & 0.0976 & 0.0126 & -0.0629 & 0.0063 \\
\hline & & ML & 0.0975 & 0.0126 & -0.0630 & 0.0064 \\
\hline & & MPL & 0.1028 & 0.0133 & -0.0672 & 0.0067 \\
\hline & & $\mathrm{EE}$ & 0.1773 & 0.0344 & -0.1153 & 0.0163 \\
\hline
\end{tabular}


Table 2: Simulation results under four different conditional mean functions with $g(Z)=\exp (-Z / 5)$. Bias: empirical bias; MSE: mean square error; CMF: conditional mean function.

\begin{tabular}{|c|c|c|c|c|c|c|}
\hline \multirow[b]{2}{*}{$N$} & \multirow[b]{2}{*}{$\mathrm{CMF}$} & \multirow[b]{2}{*}{ Estimator } & \multicolumn{2}{|c|}{$\beta_{1}^{*}$} & \multicolumn{2}{|c|}{$\beta_{2}^{*}$} \\
\hline & & & Bias & MSE & Bias & MSE \\
\hline \multirow[t]{20}{*}{200} & $\mathrm{a}$ & WR & 0.0073 & 0.0068 & 0.0001 & 0.0034 \\
\hline & & $\mathrm{AEE}$ & 0.0002 & 0.0058 & -0.0048 & 0.0037 \\
\hline & & ML & 0.0007 & 0.0059 & -0.0055 & 0.0037 \\
\hline & & MPL & 0.0019 & 0.0056 & -0.0068 & 0.0035 \\
\hline & & $\mathrm{EE}$ & -0.0045 & 0.0064 & -0.0018 & 0.0043 \\
\hline & $\mathrm{b}$ & WR & 0.0098 & 0.0057 & 0.0042 & 0.0023 \\
\hline & & $\mathrm{AEE}$ & 0.2242 & 0.0517 & -0.1459 & 0.0231 \\
\hline & & ML & 0.2241 & 0.0516 & -0.1458 & 0.0231 \\
\hline & & MPL & 0.2337 & 0.0559 & -0.1548 & 0.0257 \\
\hline & & $\mathrm{EE}$ & 0.3198 & 0.1051 & -0.2250 & 0.0538 \\
\hline & $\mathrm{c}$ & WR & 0.0063 & 0.0043 & 0.0020 & 0.0018 \\
\hline & & $\mathrm{AEE}$ & 0.0958 & 0.0098 & -0.0604 & 0.0041 \\
\hline & & ML & 0.0959 & 0.0099 & -0.0607 & 0.0042 \\
\hline & & MPL & 0.1027 & 0.0113 & -0.0663 & 0.0049 \\
\hline & & $\mathrm{EE}$ & 0.1714 & 0.0326 & -0.1093 & 0.0145 \\
\hline & d & WR & 0.0080 & 0.0061 & 0.0006 & 0.0044 \\
\hline & & $\mathrm{AEE}$ & 0.2625 & 0.0731 & -0.1784 & 0.0369 \\
\hline & & ML & 0.2622 & 0.0730 & -0.1784 & 0.0369 \\
\hline & & MPL & 0.2685 & 0.0761 & -0.1847 & 0.0389 \\
\hline & & $\mathrm{EE}$ & 0.3544 & 0.1290 & -0.2522 & 0.0677 \\
\hline
\end{tabular}




\section{REFERENCES}

Table 2 (continued): Simulation results under four different conditional mean functions with $g(Z)=\exp (-Z / 5)$. Bias: empirical bias; MSE: mean square error; CMF: conditional mean function.

\begin{tabular}{|c|c|c|c|c|c|c|}
\hline \multirow[b]{2}{*}{$N$} & \multirow[b]{2}{*}{$\mathrm{CMF}$} & \multirow[b]{2}{*}{ Estimator } & \multicolumn{2}{|c|}{$\beta_{1}^{*}$} & \multicolumn{2}{|c|}{$\beta_{2}^{*}$} \\
\hline & & & Bias & MSE & Bias & MSE \\
\hline \multirow[t]{20}{*}{200} & $\mathrm{a}$ & WR & 0.0073 & 0.0068 & 0.0001 & 0.0034 \\
\hline & & $\mathrm{AEE}$ & 0.0002 & 0.0058 & -0.0048 & 0.0037 \\
\hline & & ML & 0.0007 & 0.0059 & -0.0055 & 0.0037 \\
\hline & & MPL & 0.0019 & 0.0056 & -0.0068 & 0.0035 \\
\hline & & $\mathrm{EE}$ & -0.0045 & 0.0064 & -0.0018 & 0.0043 \\
\hline & b & WR & 0.0098 & 0.0057 & 0.0042 & 0.0023 \\
\hline & & AEE & 0.2242 & 0.0517 & -0.1459 & 0.0231 \\
\hline & & ML & 0.2241 & 0.0516 & -0.1458 & 0.0231 \\
\hline & & MPL & 0.2337 & 0.0559 & -0.1548 & 0.0257 \\
\hline & & $\mathrm{EE}$ & 0.3198 & 0.1051 & -0.2250 & 0.0538 \\
\hline & c & WR & 0.0063 & 0.0043 & 0.0020 & 0.0018 \\
\hline & & AEE & 0.0958 & 0.0098 & -0.0604 & 0.0041 \\
\hline & & ML & 0.0959 & 0.0099 & -0.0607 & 0.0042 \\
\hline & & MPL & 0.1027 & 0.0113 & -0.0663 & 0.0049 \\
\hline & & $\mathrm{EE}$ & 0.1714 & 0.0326 & -0.1093 & 0.0145 \\
\hline & d & WR & 0.0080 & 0.0061 & 0.0006 & 0.0044 \\
\hline & & $\mathrm{AEE}$ & 0.2625 & 0.0731 & -0.1784 & 0.0369 \\
\hline & & ML & 0.2622 & 0.0730 & -0.1784 & 0.0369 \\
\hline & & MPL & 0.2685 & 0.0761 & -0.1847 & 0.0389 \\
\hline & & $\mathrm{EE}$ & 0.3544 & 0.1290 & -0.2522 & 0.0677 \\
\hline
\end{tabular}


Table 3: Simulation results under four different conditional mean functions with $g(Z)=\sqrt{Z}$. SD: sample standard deviation of the parameter estimator; ESD: the average of the estimated standard deviations; CP: the empirical coverage probabilities

\begin{tabular}{|c|c|c|c|c|c|c|c|}
\hline \multirow[b]{2}{*}{$N$} & \multirow[b]{2}{*}{$\mathrm{CMF}$} & \multicolumn{3}{|c|}{$\beta_{1}^{*}$} & \multicolumn{3}{|c|}{$\beta_{2}^{*}$} \\
\hline & & SD & ESD & $\mathrm{CP}$ & SD & ESD & $\mathrm{CP}$ \\
\hline \multirow[t]{4}{*}{100} & $\mathrm{a}$ & 0.0899 & 0.0820 & 0.9550 & 0.0662 & 0.0693 & 0.9500 \\
\hline & b & 0.1068 & 0.1063 & 0.9710 & 0.0852 & 0.0896 & 0.9490 \\
\hline & $\mathrm{c}$ & 0.0911 & 0.0977 & 0.9610 & 0.0755 & 0.0812 & 0.9510 \\
\hline & d & 0.0893 & 0.0846 & 0.9700 & 0.0685 & 0.0723 & 0.9640 \\
\hline \multirow[t]{4}{*}{200} & $\mathrm{a}$ & 0.0704 & 0.0604 & 0.9740 & 0.0456 & 0.0486 & 0.9740 \\
\hline & b & 0.0664 & 0.0742 & 0.9800 & 0.0553 & 0.0626 & 0.9710 \\
\hline & c & 0.0597 & 0.0671 & 0.9700 & 0.0512 & 0.0562 & 0.9640 \\
\hline & d & 0.0729 & 0.0597 & 0.9630 & 0.0494 & 0.0502 & 0.9530 \\
\hline
\end{tabular}


Table 4: Simulation results under four conditional mean functions with $g(Z)=\exp (-Z / 5)$. SD: sample standard deviation of the parameter estimator; ESD:

the average of the estimated standard deviations; CP: the empirical coverage probabilities of the $95 \%$ confidence interval; CMF: conditional mean function.

\begin{tabular}{|c|c|c|c|c|c|c|c|}
\hline \multirow[b]{2}{*}{$n$} & \multirow[b]{2}{*}{$\mathrm{CMF}$} & \multicolumn{3}{|c|}{$\beta_{1}^{*}$} & \multicolumn{3}{|c|}{$\beta_{2}^{*}$} \\
\hline & & SD & ESD & $\mathrm{CP}$ & SD & ESD & $\mathrm{CP}$ \\
\hline \multirow[t]{4}{*}{100} & $\mathrm{a}$ & 0.1031 & 0.0968 & 0.9570 & 0.0805 & 0.0830 & 0.9480 \\
\hline & b & 0.0938 & 0.0887 & 0.9680 & 0.0712 & 0.0739 & 0.9590 \\
\hline & $\mathrm{c}$ & 0.0778 & 0.0779 & 0.9610 & 0.0590 & 0.0637 & 0.9630 \\
\hline & d & 0.1187 & 0.1165 & 0.9610 & 0.1018 & 0.0992 & 0.9310 \\
\hline \multirow[t]{4}{*}{200} & $\mathrm{a}$ & 0.0821 & 0.0720 & 0.9590 & 0.0587 & 0.0600 & 0.9470 \\
\hline & b & 0.0747 & 0.0626 & 0.9730 & 0.0479 & 0.0514 & 0.9700 \\
\hline & $\mathrm{c}$ & 0.0651 & 0.0558 & 0.9690 & 0.0419 & 0.0445 & 0.9630 \\
\hline & d & 0.0776 & 0.0833 & 0.9760 & 0.0663 & 0.0708 & 0.9640 \\
\hline
\end{tabular}


Table 5: Regression analysis results for the Bladder Tumor data: estimates of regression coefficients, estimated standard deviation (ESD), and P-values for testing

\begin{tabular}{cccccccccccc}
\multicolumn{1}{c}{$\beta_{j}^{*}=0, j=1,2,3}$. \\
\hline & \multicolumn{3}{c}{ Estimate } & \multicolumn{3}{c}{ ESD } & \multicolumn{3}{c}{ P-value } \\
\cline { 2 - 11 } & $\beta_{1}^{*}$ & $\beta_{2}^{*}$ & $\beta_{3}^{*}$ & $\beta_{1}^{*}$ & $\beta_{2}^{*}$ & $\beta_{3}^{*}$ & $\beta_{1}^{*}$ & $\beta_{2}^{*}$ & $\beta_{3}^{*}$ \\
\hline WR & 0.6178 & -0.6060 & 0.5010 & 0.2067 & 0.2117 & 0.5056 & 0.0028 & 0.0042 & 0.3217 \\
AEE & 0.6354 & -0.7656 & 0.0997 & 0.2027 & 0.3486 & 0.4380 & 0.0017 & 0.0280 & 0.8200 \\
ML & 0.6380 & -0.7644 & 0.0973 & 0.2184 & 0.3242 & 0.3980 & 0.0035 & 0.0180 & 0.8100 \\
MPL & 0.4211 & -0.6284 & 0.2146 & 0.1577 & 0.2811 & 0.2913 & 0.0076 & 0.0250 & 0.4600 \\
EE & 1.0306 & -1.2630 & 0.3681 & 0.3408 & 0.4795 & 0.5730 & 0.0025 & 0.0084 & 0.5200 \\
\hline
\end{tabular}

